\title{
David M. Smith et l'invention de la pince à linge dans l'Amérique du milieu du XIXe siècle
}

\author{
David M. Smith and the invention of the clothespin in mid-19th century
} America

\author{
Philippe Bruyerre ${ }^{1}$ \\ ${ }^{1}$ Docteur en histoire des techniques EHESS/CAK
}

\begin{abstract}
RÉSUMÉ. La pince à linge, un objet technique banal, a été brevetée en 1853 par David M. Smith, inventeur et entrepreneur de la petite ville de Springfield dans le Vermont. A partir de sa biographie, l'article examine les déterminants techniques et socio-économiques d'un objet technique qui a fait l'objet de plus de 2000 brevets en un siècle et demi aux Etats-Unis et constitue une icône de l'inventivité américaine.

ABSTRACT. The clothespin, an everyday technical object, was patented in 1853 by David M. Smith, an inventor and entrepreneur from the small town of Springfield, Vermont. Based on his biography, this article examines the technical and socio-economic determinants of an object that has been the subject of more than 2,000 patents in a century and a half in the United States, and is an icon of American ingenuity.

MOTS-CLÉS. Pince à linge, invention, entrepreneur, Vermont, icône.

KEYWORDS. Clothespin, invention, entrepreneur, Vermont, icon.
\end{abstract}

\section{Introduction}

Les objets du quotidien sont des objets souvent oubliés par les historiens des techniques qui leur préfèrent des objets plus emblématiques [ROC 97]. Parmi les choses banales qui nous entourent, la pince à linge est un objet phare par sa simplicité et sa nouveauté radicale quand elle apparait au milieu du XIXe siècle. Le crédit de cette invention est attribué à David M. Smith (1809-1881) de la petite ville de Springfield dans le Vermont aux Etats-Unis.

Moins connu que Linus Yale (New Hampshire) et sa serrure de sûreté, Samuel Colt (Connecticut) et son revolver ou Thomas Blanchard et son tour d'usinage (Massachusetts), David M. Smith est un entrepreneur et inventeur particulièrement prolifique. Installé à Springfield (Vermont), il crée avec d'autres inventeurs locaux différentes entreprises qui fabriquent des pinces à linge, des porteserpillières, des manches de brosse, des agrafes pour manteaux... des produits de base (basic commodities) de l'économie domestique, des «commodités». Il participe ainsi activement au processus de «mécanisation de la vie quotidienne » [GIE 80] qui se met en place dans la seconde moitié du XIXe siècle et fait émerger une culture matérielle constitutive de la nation américaine.

L'histoire de cet objet est abordée suivant le double point de vue de la biographie de son inventeur D. M. Smith et du contexte économique et social de l'Amérique de la seconde moitié du XIXe siècle dans lequel l'entrepreneur se situe. Cependant, cette approche laisse de côté l'objet technique « pince à linge » qui se constitue au travers des interactions entre améliorations techniques et intégration dans l'économie domestique dans une dimension symbolique, voire iconique. Toutes les améliorations envisageables ne sont ni possibles pour les inventeurs et entrepreneurs, ni souhaitées par les usagers : l'objet technique, de par son existence, joue un rôle central dans l'innovation et l'entrepreneuriat. Si en suivant Jablonka «faire de l'histoire en sciences sociales, c'est comprendre ce que les gens font » [JAB 14], ce que les gens font, innovateurs/entrepreneurs et usagers, n'est compréhensible que dans leur contexte d'action au centre duquel se trouve l'objet technique. 


\section{David M. Smith, inventeur et entrepreneur vermontois}

\subsection{Des origines rurales dans le Vermont}

La biographie de David M. Smith peut être retracée à partir d'ouvrages de chroniqueurs locaux et de généalogistes parus à la fin du XIXe siècle comme ceux d'Horace Hubbard et Justus Dart [HUB 95] pour Springfield et de Silvanus Hayward [HAY 81] pour Gilsum ainsi que le récit plus récent de Frederick Richardson [RIC 00]. Ces sources permettent de retracer dans ses grandes lignes l'itinéraire de cet homme au nom banal, Smith, (le nom des «sans-nom » à leur arrivée en Amérique), résidant à Springfield (52 villes portent ce nom aux Etats-Unis, et Springfield est élue en 2007 lieu de résidence des Simpson, l'archétype de l'Américain moyen contemporain [SEC 07]).

David McAllister Smith naît le 29 mai 1809 à Hartland dans l'Etat du Vermont (comté de Windsor). Il est le cadet d'une famille de douze enfants, sept filles et cinq garçons, issus de l'union de Samuel Smith et de Margaret Wright. Orphelin de mère à l'âge de 6 ans, il est pris en charge par ses trois sœurs ânées, âgées de 25 à 30 ans. Les conditions de vie sont difficiles sur une terre marécageuse avec des ressources limitées aux revenus modestes de son père, cordonnier et pensionné de l'armée.

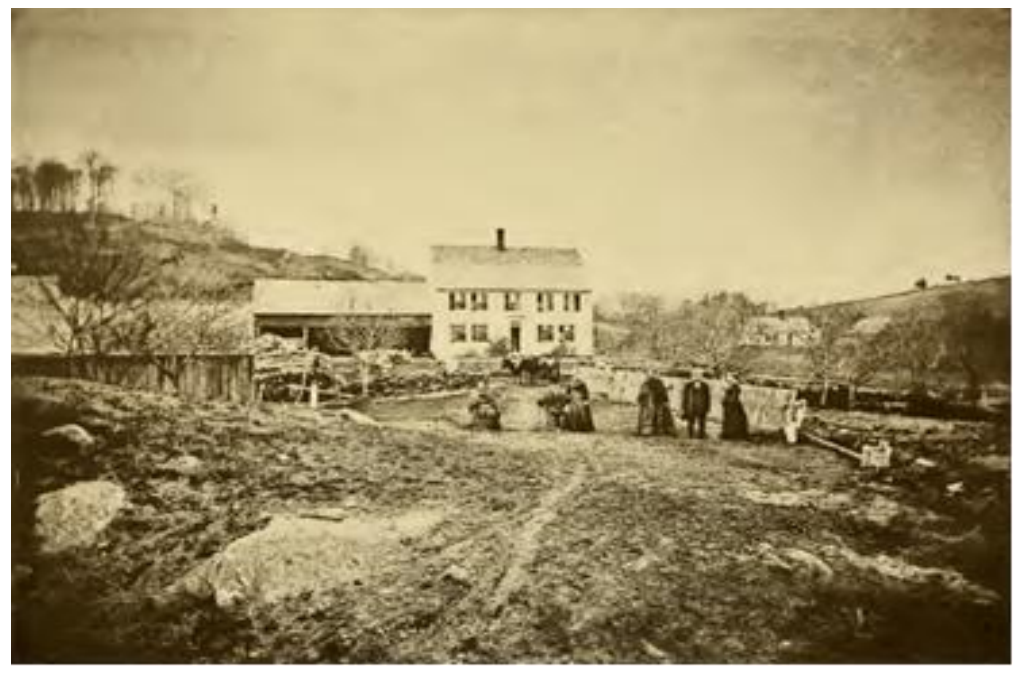

Figure 1. Gilsum [HAY 81].

En 1821, la famille déménage pour Gilsum (New Hampshire), à 60 kilomètres au sud d'Hartland, où D.M. Smith commence son apprentissage de charpentier jusqu'en 1828. Il travaille ensuite avec Luther S. Hemenway dans un atelier de fabrication de manches de poinçon inventé par ce dernier en 1828. D.M. Smith dépose ses deux premiers brevets sur ce type de manche en 1832 et 1836 [SMI 32]. Malgré ses faibles ressources, il lui est possible d'obtenir ces brevets car leur coût est faible aux Etats-Unis, 30 dollars en 1836 à une époque où un charpentier gagne environ 1,5 dollar par jour [BUR 29]. En 1869, il en coûtera 35 dollars à comparer aux 75 dollars en Grande Bretagne et 300 dollars en France [USP 69] même en ajoutant à ce coût celui du conseiller juridique, d'environ 25 dollars [MUN 64].

En 1831, il se marie avec la sœur aînée de son associé, Lucy Barker Hemenway et habite près de l'atelier. Père de 5 enfants, il s'investit dans la vie locale : il représente Gilsum au Parlement du Vermont en 1840-1841. Il participe à la société littéraire ou Lyceum en 1833-1834 et fait partie du comité à l'origine de la Société anti-esclavagiste de Gilsum.

\subsection{Le tournant des années 1840}

Les années 1840 sont celles des roaring forties aux Etats-Unis caractérisées par une extension territoriale phénoménale (triplement de la surface en 50 ans), un recentrage sur le marché intérieur lié à la deuxième guerre d'Indépendance de 1812 qui limite les débouchés à l'exportation, et une démographie galopante. Le mythe agrarien de la société coloniale, celui de la révolution jeffersonienne, s'éloigne. C'est aussi l'époque du second Grand Réveil, avec ses prédicateurs et des 
camp meetings, réunions festives de plein air exprimant la ferveur évangélique, des sectes religieuses [WAR 80] émergent pour surmonter une Amérique fondée sur les divisions et les conflits, adventistes de Miller (1832), shakers d'Ann Lee, unitariens de Chenning (1819), Mormons et Saints des Derniers Jours de Joseph Smith dont la mère Lucy Mack Smith est née à Gilsum, mouvement transcendentaliste initié par Emerson...

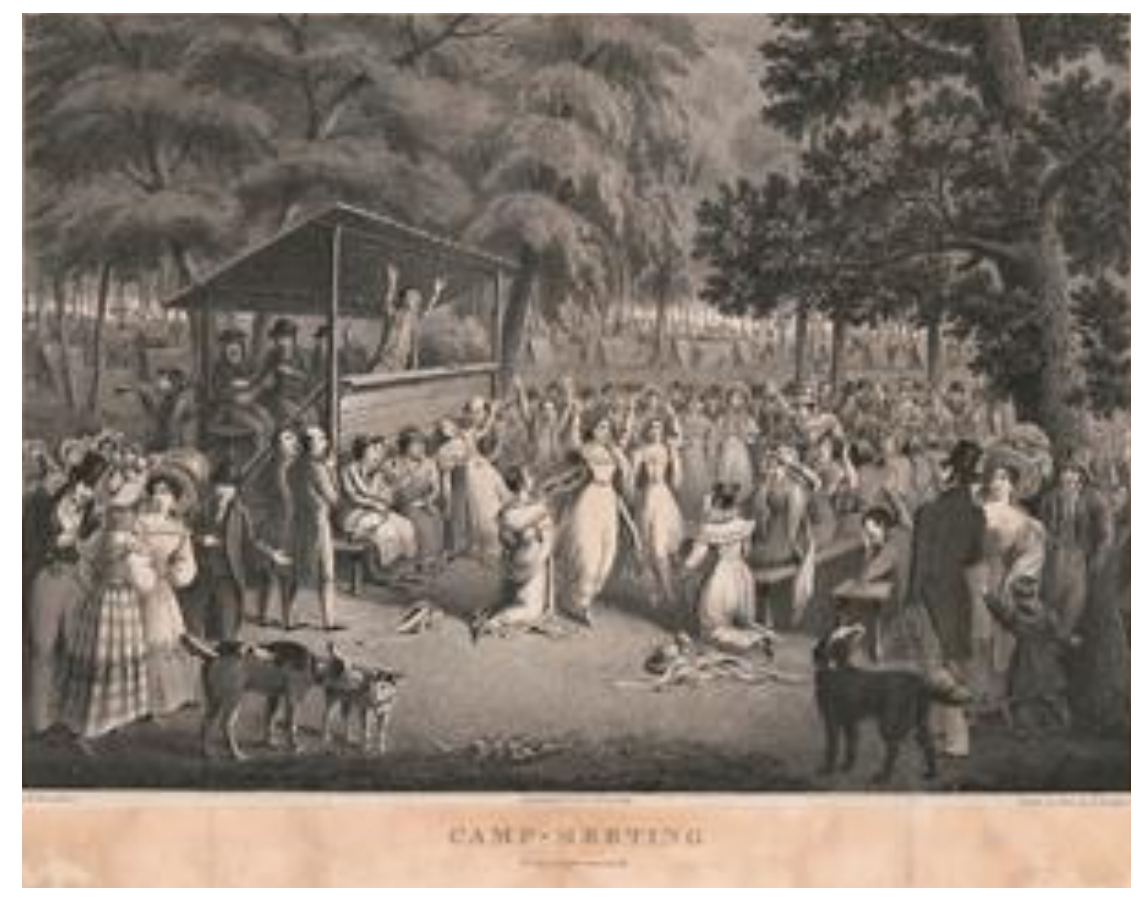

Figure 2. Camp meeting en 1829 [RID 29].

D.M. Smith se trouve au cœur de cette effervescence religieuse. Quand il est encore à Gilsum, il fait en effet partie de la Christian Church créée à l'initiative des pasteurs Edward B. Rollins et John Smith, son frère aîné de 11 ans de plus que lui et prêchant une doctrine proche de l'hérésie selon le Révérend A. J. Howard. Sa belle-famille, les Hemenway, initialement baptiste, puis influencée par Elder Rollins adhère au mormonisme et part en Utah en 1847 [THE 20]. De fait, les trajectoires d'Hemenway et de Smith, tous deux inventeurs et entrepreneurs, l'un rejoignant l'Utah des Mormons, le second partant pour Springfield dans la création d'entreprise sont les deux faces d'une même identité américaine. «L'Union est une partie de la religion de ce peuple», disait Emerson en 1851 [EME 12]. Hemenway et Smith répondent chacun à leur manière au malaise qui se développe dans un monde de plus en plus matérialiste et de plus en plus violent où l'argent prend une place de plus en plus importante comme le dénonce Henry David Thoreau dans Walden ou la vie dans les bois en 1854 [THO 54].

Dans ce contexte, les femmes jouent un rôle central et revendiquent leur place dès les années 1820 . Elles cherchent leur émancipation sans pour autant contester la domination masculine. Ce mouvement s'affirme dans les années 1840 avec les ouvrages de Catherine E. Beecher [BEE 43] et de Margaret Fuller de 1845 [FULL 45] ainsi que la Déclaration de Sentiments de 1848. Elles militent notamment contre l'esclavagisme, le ferment de la Guerre civile qui éclatera en 1861, et contre l'alcoolisme (sociétés de tempérance).

L'émancipation se manifeste aussi avec le recrutement de jeunes filles du monde rural embauchées par les filatures. Ces ouvrières sont des jeunes filles d'origine rurale de 15 à 35 ans, qui partent à Lowell ou Chicopee (Massachussetts) travailler dans les filatures de coton dans les années 1820-1850. Le Waltham-Lowell system sera mis en place pour loger ces filles d'usine (factory girls), moins payées que les hommes, travaillant de 11 à 13 heures par jour dans le bruit et la promiscuité, mais conquérant leur autonomie financière, soutenant leurs parents, apprenant à lire et à écrire, s'émancipant en somme. Dans leur propre journal Lowell Offering de 1840, certaines de ces factory girls vantent les plaisirs de la vie d'atelier qui, dans la répétitivité des gestes, laisse place à la réflexion sur d'autres sujets [SGB 40]. La question de l'émancipation féminine rejoint celle de la démocratie car celles-ci sont absentes de la Constitution de 1776, comme d'ailleurs les Noirs et les Indiens. Les pères fondateurs ont oublié les 
« mères fondatrices » [LAC 18] qui vont lutter avec obstination dans la seconde moitié du XIXe siècle pour obtenir des droits civiques (droit de vote obtenu en 1920) et des responsabilités politiques.

\subsection{Inventeur entrepreneur à Springfield}

L'année 1842 constitue un tournant dans la vie de D.M. Smith : il s'installe à Springfield (Vermont), à 30 kilomètres de Gilsum, une bourgade de 2600 habitants située dans la vallée de la Black River sur une série de cascades avec une dénivelée de 35 mètres sur moins de 200 mètres [COOL 59]. Du point de vue naturel, le Vermont - les Montagnes Vertes - est un pays de forêts qui représentent environ $90 \%$ de sa surface en 1760 et furent exploitées à un tel point par les «forestiers » ou Pionniers qu'elles ne couvraient plus que $38 \%$ en 1905. Elles fournissent du bois en quantité, matière première de nombreuses activités : bûcheronnage et sciage, fabrication d'objets et de pièces de mobilier en bois [VER 10].

A cette première vague de forestiers, succède une vague de mécaniciens au sens large qui créent des barrages et installent des scieries hydrauliques comme William Lockwood en 1774, puis des ateliers de fabrication utilisant la force hydraulique. L'énergie hydraulique est et restera longtemps la première source d'énergie de l'industrie manufacturière [HUN 79], à Springfield comme dans le Vermont. En 1900 à Springfield, la puissance installée pour les activités manufacturières est encore de de 87515 CV pour l'hydraulique contre $45142 \mathrm{CV}$ pour les machines à vapeur et $729 \mathrm{CV}$ seulement pour l'électricité [ANO 04].

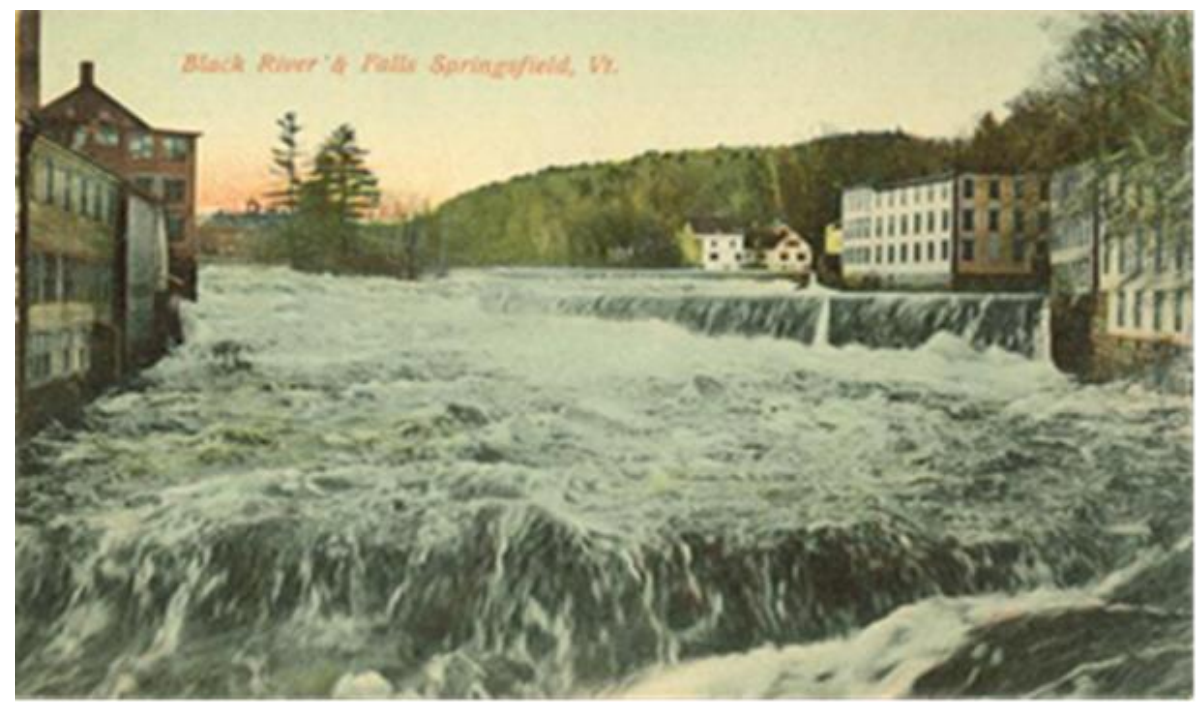

Figure 3. La Black River et les chutes à Springfield vers 1910 [BLA 10].

La population du Vermont connait une croissance importante au début du XIXe siècle, passant de 85416 habitants en 1790 à 228713 en 1820 [TUC 43]. C'est la seconde frontière au sens de Turner pour qui « le Vermont peut être considéré comme l'apogée du mouvement de la frontière » [TUR 20]. Dans des conditions de frontière Est-Ouest, les Vermontois deviennent, sous la pression des conditions naturelles et des conflits territoriaux avec les Français et les Indiens, des pionniers de la démocratie, menant une vie simple et auto-suffisante.

A son arrivée à Springfield, D.M. Smith commence par exercer différents métiers (mécanicien, orfèvre, plaqueur bois) avant de s'associer en 1843 avec le docteur Eleazer Crain, inventeur de ceintures abdominales [CRA 43]. Sa femme Lucy décède en 1847 et il se remarie l'année suivante avec Sarah G. Burr, fille d'Asahel Burr de Springfield. En parallèle, il dépose différents brevets : serrure de porte (1846), procédé de trempage de l'acier (1848), machine à coudre (1850) [SMI 46]. Une de ses inventions est une serrure à combinaison (1849) qu'il fabrique avec Ira et Isaac G. Davis, dans leur atelier de fabrication de boucles de chaussures. Cette serrure est jugée par l'expert Alfred C. Hobbs comme la seule ne pouvant être fracturée, mais la concurrence est rude notamment avec Linus Yales Jr., et ses « serrures magiques brevetées » (Patent Magic Locks) dès 1851 (serrure de sûreté). En 1854, 
il brevète le premier toc pour tour [SMI 54] pour l'usinage de pièces longues et vend le brevet pour 900 dollars.

D.M. Smith obtient en 1853 un brevet pour une « pince à ressort pour corde à linge » (spring-clamp for clothes line) [SMI 53]. La pince à linge mécanisée que propose Smith arrive dans une société qui se recentre sur son espace domestique sous la direction de la femme américaine, la «femme républicaine » [KER 76], dont le mari pionnier soit travaille dur, soit fait la guerre. De nombreuses inventions de l'époque concernent la «mécanisation des tâches ménagères » [GIE 80], notamment la blanchisserie avec les premières machines à laver, les fers à repasser, les étendoirs et les pinces à linge. Avec cette dernière, la bonne tenue du foyer s'affiche avec un linge bien étendu sur une corde à linge extérieure et bien maintenu par le ressort des pinces. L'extimité de l'étendage renvoie à l'intimité de la maison, exposant à la vue de tous l'ordre et la propreté de l'espace intérieur, générant un sentiment de fierté et de confiance en soi. Le placement du linge sur la corde à linge, le choix des pinces à linge renvoient à tout un rituel de gestes mécaniques, d'émotions, de petits plaisirs et d'agacements toujours actuels [KAU 97].

La même année, il crée son entreprise, D.M. Smith \& Co avec Albert Brown, Hamlin Whitmore et Henry H. Mason, des entrepreneurs locaux, pour fabriquer ces pinces à linge. L'entreprise emploie 60 femmes (girls) et semble connaître un succès foudroyant avec un chiffre d'affaires de 40000 dollars par an en 1853, ce qui en fait le second entrepreneur de la ville. Selon le chroniqueur Hubbard, la mise de fonds est faible, cinq cents dollars et les profits (l'année n'est pas précisée) s'élèvent à 72000 dollars.

En 1856, il s'associe avec Joel A.H. Ellis, Hamlin Whitmore, Henry H. Mason et Albert Brown pour fabriquer un panier de ferme inventé par Ellis [ELL 57]. Cette entreprise est un échec. Il continue son activité d'inventeur avec un plantoir et une règle pour charpentier en 1859 ainsi qu'une agrafe métallique pour vêtement dite «à crochet et œillet 》 (hook and eye) en 1860 [SMI 59]. Cette dernière invention est un succès, les affaires sont florissantes avec la demande croissante de l'armée en ces années de Guerre civile et l'augmentation des taxes douanières sous la présidence des Républicains du Nord [SCH 94]. En 1859, avec un capital de 20000 dollars, il est le troisième entrepreneur de la ville d'après Coolidge [COOL 59]. Image de cette prospérité, D.M. Smith construit en 1858 une grande maison de plus de $300 \mathrm{~m}^{2}$ de style Greek Revival en bas de la rue principale (128 Main Street), édifice aujourd'hui classé [SPR 83a]. L'atelier, initialement installé sur la rive ouest de la rivière, emménage en 1860 dans de nouveaux locaux proches de sa maison. 


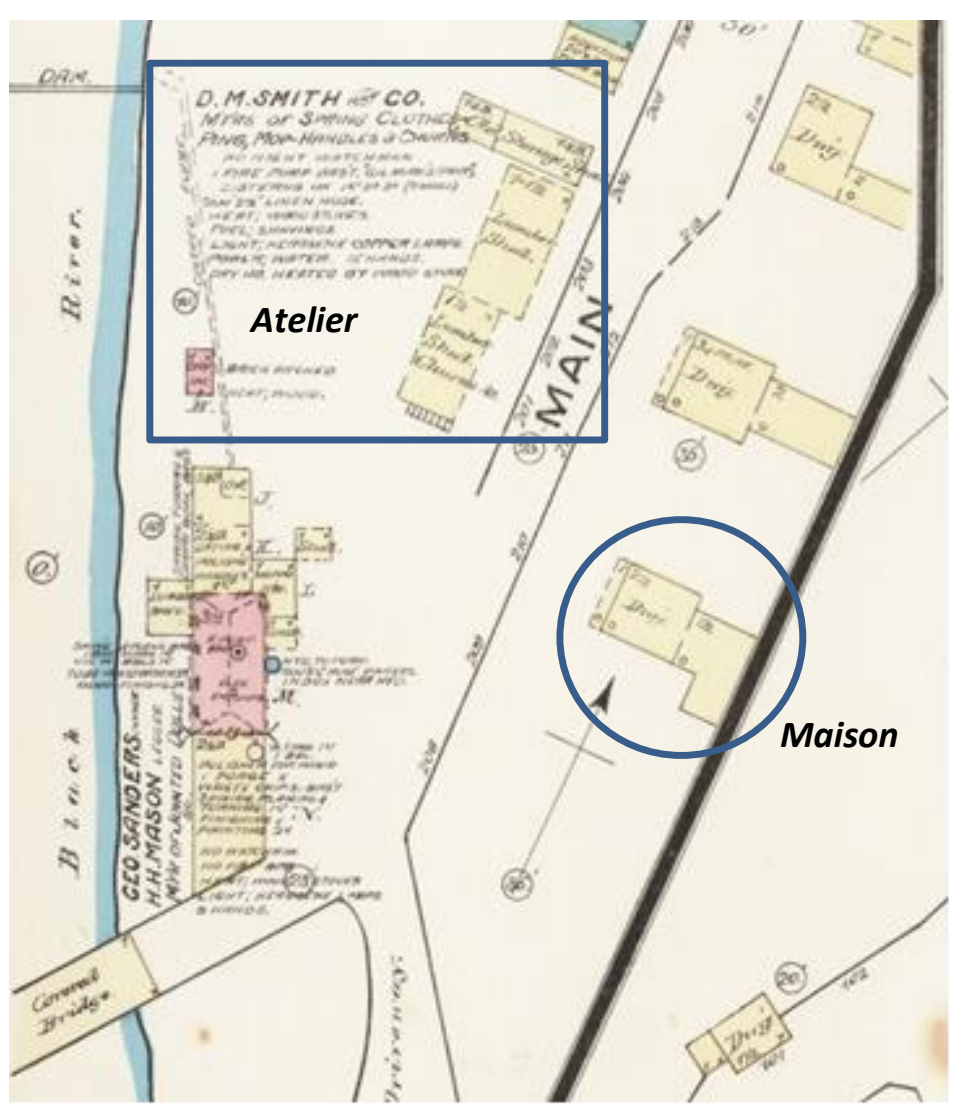

Maison et atelier de D.M. Smith, 1885 [SAN 85]

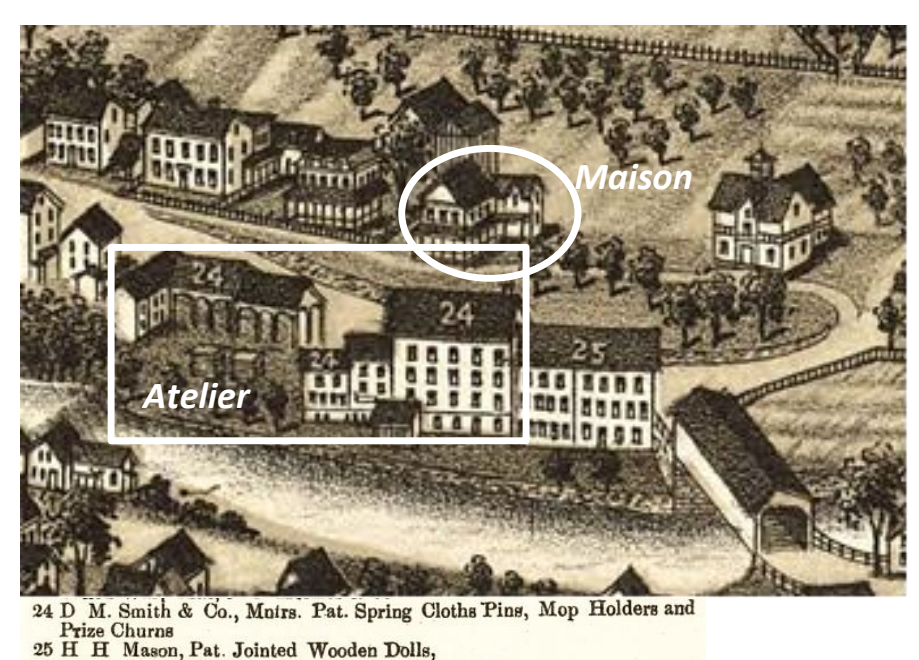

Springfield en 1886 [BURLE 86]

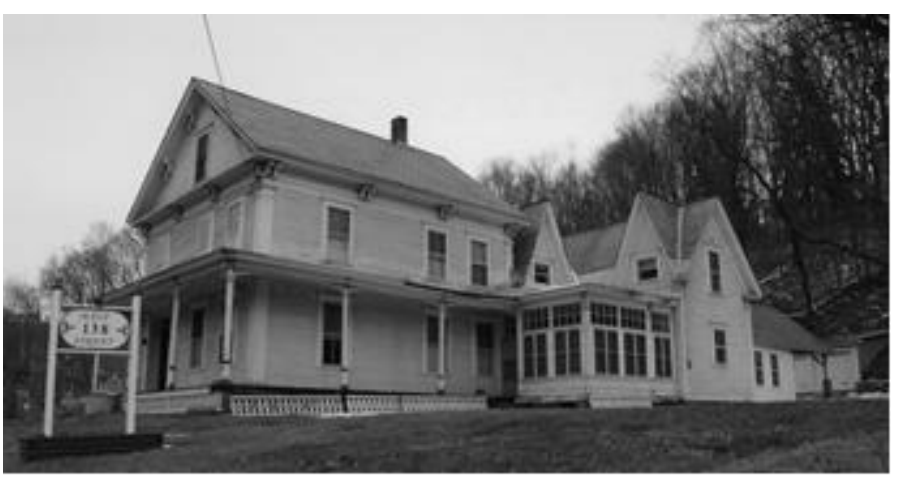

Vue de la maison de D.M. Smith, 1983 [SPR 83b]

Figure 3. Maison et atelier de David M. Smith à Springfield.

Cet atelier continue la fabrication de pinces à linge et d'agrafes pour vêtement. En 1864, une nouvelle entreprise Smith, Mason \& Co, est créée avec Albert Brown, Henry H. Mason, and Alvin C. Mason. Cette entreprise ajoute aux pinces à linge et aux agrafes la fabrication de manches de balais, de brosses. D.M. Smith conjugue activité entrepreneuriale et activité inventive (nouveau brevet d'agrafe en 1863, porte-papier en 1866 [SMI 63]) et développe de nouvelles pinces à linge en 1867 et en 1874 [SMI 67].

Les inventeurs entrepreneurs de Springfield, les Smith, Mason, Ellis, Whitmore... forment un groupe orienté plus vers la communauté que vers la nation avec des activités religieuses (huit églises de confessions différentes à Springfield), philanthropiques (bibliothèques, écoles, société de tempérance contre les ravages de l'alcoolisme), politiques (anti-esclavagisme). Tous ces acteurs se connaissent par les affaires qu'ils font ensemble, par les lectures en commun de la Bible, par les associations qu'ils soutiennent ; ils partagent ces activités avec leurs employés, au-delà des clivages de classe ou d'origine. Par ailleurs, ces entrepreneurs exportent au travers de marchands et d'agents indépendants et n'ont pas de véritables contacts avec le client final [NYE 98].

D.M. Smith ne participe pas à la Guerre civile de 1861 puisqu'il est âgé de plus de 45 ans, mais est durement touché avec la perte de son fils Laforest Marcellus en 1865, alors âgé de 32 ans. D.M. Smith s'associe en 1868 avec Henry C. Burr, son beau-frère et Isaac B. Smith pour la fabrication de colliers pour chevaux pour créer la société Smith, Burr \& Co qui devient The Vermont Hame Company avant de cesser son activité en 1875. Cette même année, âgé de 66 ans, il cède son entreprise à Albert Brown, que ce dernier revend à $\mathrm{H}$. Mason en 1877. L'atelier emploie 15 à 20 ouvriers et fabrique environ 
5000 gross $(1$ gross $=$ douze douzaines $=144)$ de pinces à linge et 600 gross de têtes de serpillère par an ainsi que des manches de brosse et des barattes de marque Prize, des poupées articulées en bois, des jougs pour bœufs [ALD 69].

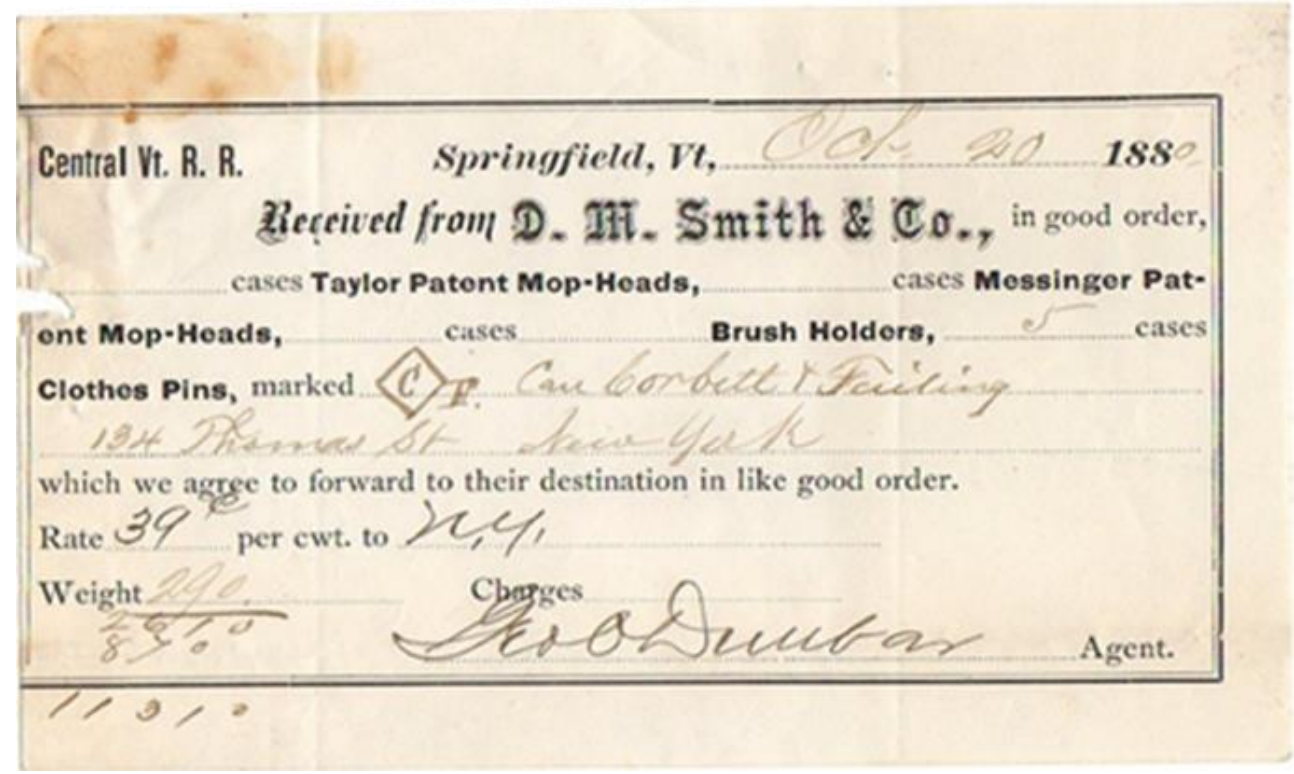

Figure 4. Bordereau d'expédition par train pour le magasin Corbett \& Failing de New York en 1880.

La société D.M. Smith \& Co cesse ses activités en 1887. L'année suivante, un groupe d'hommes d'affaires de Springfield installe sur ce terrain l'entreprise Jones et Lamson Machine Tool qu'ils vont chercher à Windsor et font venir un directeur, James Hartness, inventeur prolifique titulaire de plus de cent brevets [ROE 26]. C'est une nouvelle ère pour Springfield qui passe de l'atelier mécanisé à l'entreprise industrielle de fabrication de tours d'usinage. Springfield devient alors la « Vallée de la précision » avec la Fellows Gear Shaper Company (1896), la Lovejoy Tool Company (1917).

David M. Smith s'éteint le $1^{\text {er }}$ novembre 1881. En guise d'épitaphe, Levi K. Fuller écrit en 1894 : «L'accumulation de richesse n'était pas son objectif et il ne récolte guère le fruit de ses inventions. Il était aussi un homme d'esprit et poète et l'histoire de son village abonde de ses anecdotes. » [FULL 94].

\section{L'objet technique "pince à linge "}

Dans le cycle du lavage du linge, la phase de séchage est la plus simple, mais la moins connue du point de vue de l'histoire des techniques. Au Moyen-Age, on trouve des figurations d'étendage de linge à même le sol d'un pré, sur les buissons ou des rochers mais aussi sur des barres de bois installées entre deux poteaux préfigurant la corde à linge comme sur la représentation ci-dessous de 1531. Sur de nombreuses peintures de la fin du XIXe siècle et du début du XXe, la pince à linge est absente, le linge étant simplement posé sur le fil. Dans la description qu'en donne Marie-Cécile Riffaut, le séchage du linge dans les années 1900 reste un processus entièrement manuel, le seul équipement étant la corde à linge :

«Dès qu'une corbeille de linge rincé était remplie, elles [les laveuses] allaient à deux en étendre le contenu sur les rangées de fils de fer suspendus à des piquets dans le pré annexe. Il y avait aussi un petit hangar insuffisant pour les jours de pluie. Le soir, la voiture attelée venait reprendre le linge sec et contre-plié (plis de séchage). » [RIF 80] 

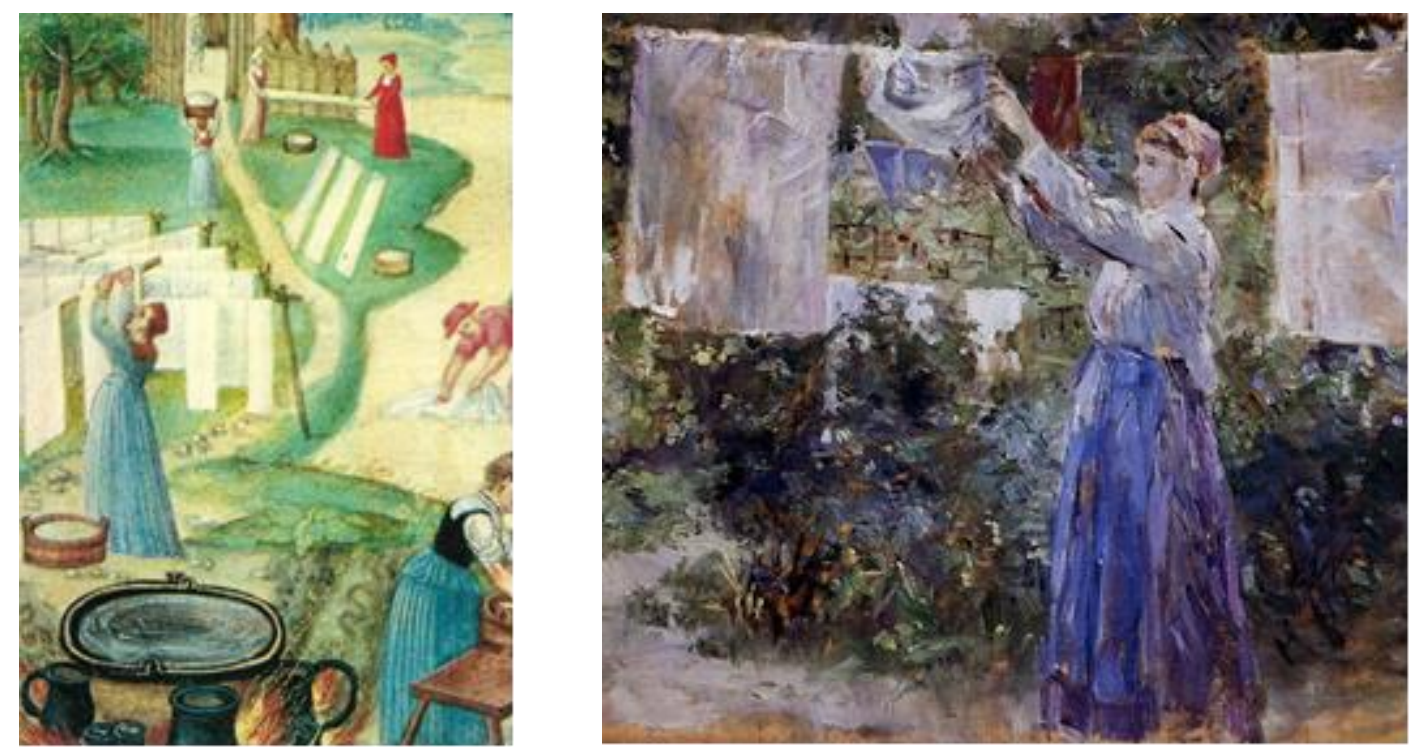

Figure 5. Etendage du linge : 1531 (à gauche) [ANO 31] et 1881 (à droite) [MOR 81].

\subsection{De la broche à la pince}

$\mathrm{Au}$ XVIIe siècle, ou peut-être avant, apparaissent des épingles à linge (clothes pin aux Etats-Unis), appelées aussi broches à linge (clothes peg au Royaume-Uni), des morceaux de bois fendus sur leur longueur et maintenus par une bande de métal. Certains historiens américains affirment que la broche à linge aurait été inventée par les Shaker, des puritains issus des Quakers [MIL 10] [WEL 19], mais il semble plus probable que la broche à linge ait été apportée d'Angleterre par les colons selon Barbara Suit Janssen, du Musée national d'histoire américaine [GRE 12].

La pince à linge vient apporter deux nouveautés comme l'explique David M. Smith dans son brevet de 1853. D'abord, « cet instrument, contrairement à la broche à linge en bois d'usage courant, n'use pas les vêtements ou ne les marque pas lorsqu'il est utilisé. » Ensuite, «un autre avantage très important est qu'elle ne peut pas être détachée des vêtements par le vent. » Absence de marquage du linge et résistance au vent sont les deux moteurs de l'innovation de la pince à linge. L'idée n'est nouvelle puisqu'un certain Samuel Prior avait déposé un brevet pour ce type de dispositif en 1832 [PRI 32].

Tous les composants de base de la pince à linge telle que nous la connaissons aujourd'hui sont présents dans le brevet de 1853 de D. M. Smith : les mâchoires pour serrer le linge sur le fil, les deux bras pour ouvrir les mâchoires, l'articulation centrale des deux bras et le ressort (de compression) de maintien en position fermée. Les deux autres brevets de 1867 et 1874 reprennent ces mêmes composants, cherchant à améliorer l'articulation centrale en remplaçant le fil de fer par un axe en bois. D.M. Smith en bon gestionnaire de son portefeuille de brevets, modifie un composant pour justifier de la nouveauté et protéger son activité car la concurrence est rude dans le secteur de la pince à linge. Et en bon entrepreneur, il améliore sa fabrication en tenant compte du retour d'expérience des usagères. L'innovation se construit par amélioration et retour d'expérience en modifiant la conception et en adaptant le process de fabrication pour répondre aux demandes de meilleure résistance et de moindre marquage du linge par les usagères.

Le succès de Smith attire l'intérêt et rien qu'à Springfield (Vermont) cinq brevets [ALD 52] sont déposés dont deux reprennent une structure comparable (mâchoires, ressort...). De 1842 à 1887, quelques 146 brevets de pince à linge sont déposés aux Etats-Unis [LAH 07]. 


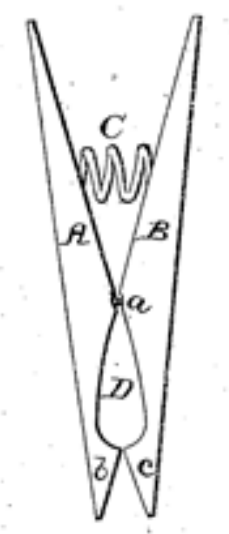

Smith, 1853.

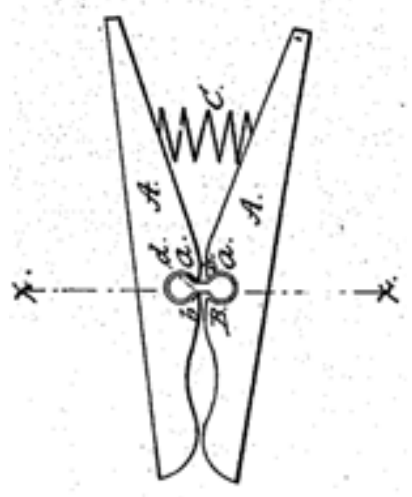

Smith, 1867.

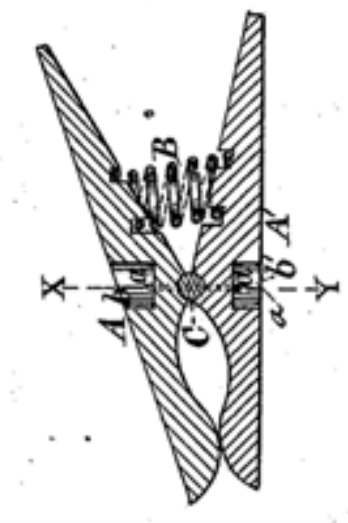

Smith, 1874.

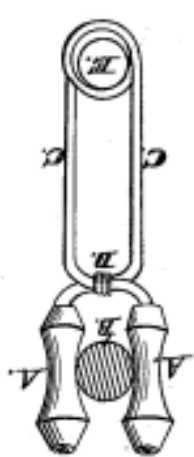

Aldrich, 1852

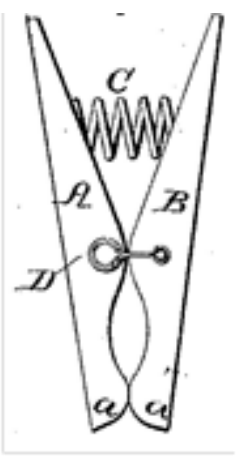

Johnson, 1867

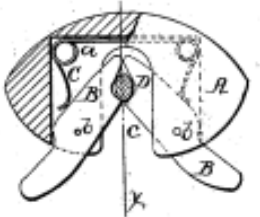

Boutell, 1867

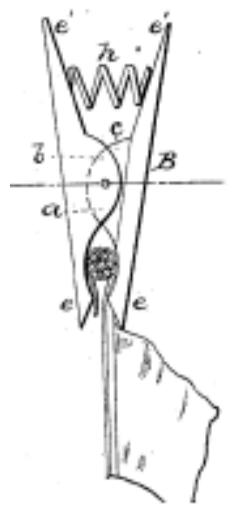

Britton, 1867

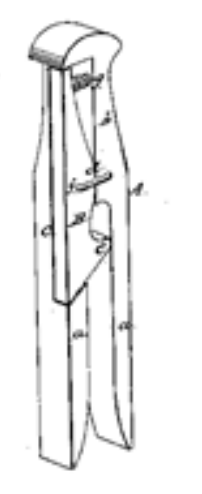

Taylor, 1868

Figure 6. Brevets de pince à linge : les trois brevets de David M. Smith [SMI 53], [SMI 67] et ceux d'inventeurs de Springfield [ALD 52].

Par la suite, un autre inventeur du Vermont, Solon E. Moore brevète en 1887 une pince à linge [MOO 87] dont la particularité est de faire jouer au ressort le rôle d'articulation en utilisant un ressort de torsion, et non pas de compression comme pour D.M. Smith. Cette pince à linge est fabriquée dans la capitale du Vermont, Montpellier, par la United States Clothespin Co.

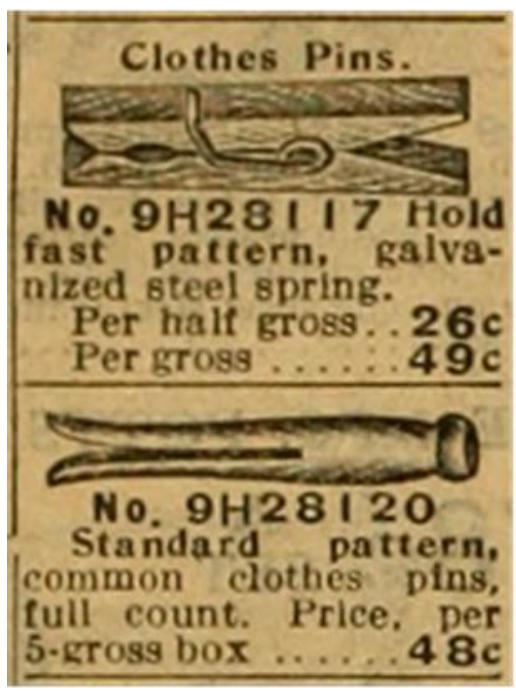

Figure 7. Pinces à linge du catalogue Sears de 1912 [SEA 12]. 
La pince à linge «mécanisée » de D.M. Smith ou de Solon E. Moore ne détrône cependant pas la broche à linge. En 1912, le grand magasin Sears propose des pinces à linge à ressort Hold fast fastener («l'attache qui tient bon ») avec ressort en acier galvanisé à 49 cents la gross (144 pinces à linge) alors que les broches à linge Standard pattern (modèle standard de type broche) valent 48 cents la boîte de cinq gross, un prix cinq fois inférieur.

La fabrication des premières broches à linge est manuelle. Ces broches sont fabriquées soit à la maison lors des veillées d'hiver, soit par des gitans nomades dans leur roulotte comme en Angleterre. George Smith évoque en 1880 la fabrication de ces broches à linge et donne une figure d'un atelier familial de gitans [VES 44] [MAY 81], une sorte de proto-industrie nomade [SMIG 80], organisée en postes de travail (écorçage en plongeant les morceaux de bois dans l'eau chaude, biseautage des branches, découpe de la bande de métal et assemblage). Ces broches à linge sont aussi fabriquées par de jeunes garçons à l'aide d'un simple canif, la figure du whittling boy évoqué en 1857 par le Révérend John Pierpont [PIE 57] et représenté dans le tableau de Winslow Homer de 1873.
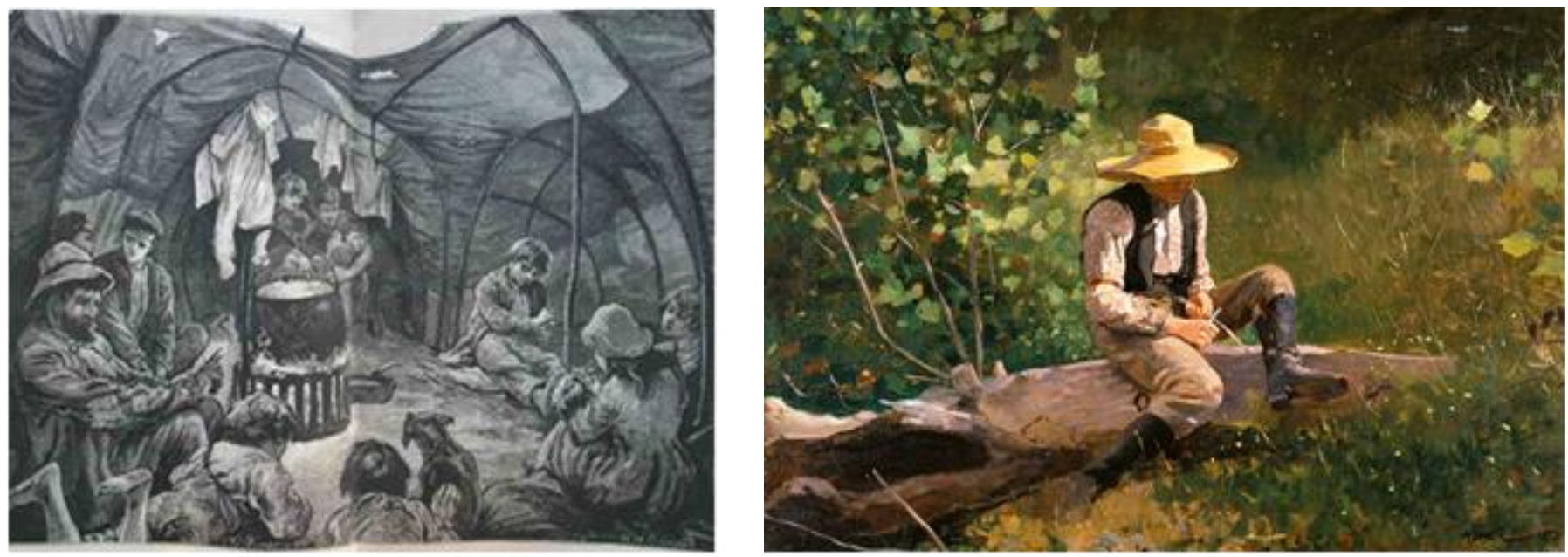

Figure 8. Fabrication manuelle de broches à linge par les gitans [SMIG 80]. A droite : "The Whittling Boy » de Winslow Homer, 1873 (1836-1910).

A la création de l'entreprise D.M. Smith, la fabrication est artisanale, assistée de machines à bois simples (scies, toupies, tours) et organisée de façon similaire à celle des gitans. Les branches doivent être de plus percées au point d'articulation pour recevoir un fil de fer qui est ensuite torsadé pour réaliser l'articulation centrale. Le ressort, enroulement de fil de fer, est placé sur les branches dans des lamages. Les pinces à linge sont ensuite emballées dans des boîtes en bois, réalisées sur place, prêtes à être expédiées vers le magasin de vente jusqu'à New York par train, le chemin de fer de la Central Vermont Railways étant ouvert en 1849.

En 1859, le processus de fabrication est mécanisé par Alvin C. Mason, associé de D.M. Smith dans Smith, Mason \& Co, qui dépose le brevet d'une machine à sertir les pinces à linge [MAS 59]. Cette machine, relativement complexe, mécanise l'assemblage de la pince à linge : les deux branches ainsi que les fils de fer qui constituent l'articulation sont placés dans cette machine entraînée par la force hydraulique. Deux pinces viennent saisir le fil de fer et le torsadent pour obtenir la pince à linge (en bas à gauche sur la figure ci-dessous).

Cette machine est conduite par de jeunes femmes (quand les chroniqueurs parlent du nombre d'employés, il s'agit de girls), une main d'œuvre disponible et moins chère, jugée plus rapide et précise que les hommes dans ce travail répétitif. Les conditions de travail sont dures dans le bruit, la poussière de bois et le danger de ces engrenages non protégés, comme le décrit un journaliste en 1915 [ANO 15]. La cadence de fabrication est importante, la valeur ajoutée aussi comme le remarque un observateur en 1900: «Un rondin de bois coûtant 2 dollars permet d'obtenir 12000 pinces à linge d'une valeur de près de 100 dollars. » [ANO 00] 


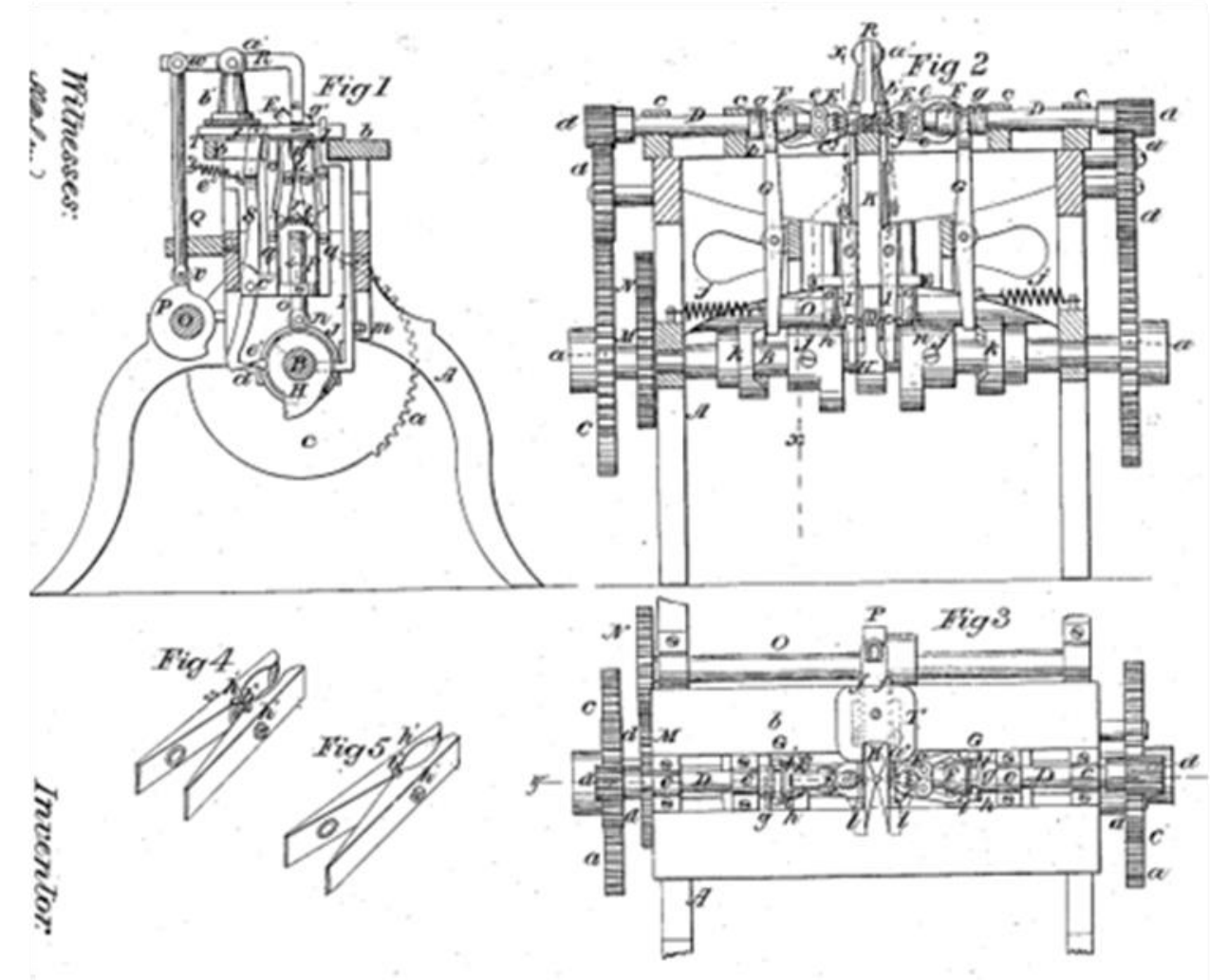

Figure 9. Machine d'assemblage de pinces à linge. [MAS 59].

Pour la fabrication des agrafes, D.M. Smith avait déjà inventé une machine spéciale que Levi K. Fuller présente en 1894 comme une «une merveille d'ingénuité » qui prend le fil d'une bobine, le cintre pour réaliser la boucle. La machine compte les boucles, les place sur un carton et emballe dans une caissette en bois prête à la commercialisation. Elle coûte 20000 dollars. [FULL 94]

Ce qu'apporte cette mécanisation, c'est non seulement une production accrue et un prix de revient plus faible, mais c'est aussi une plus grande uniformité du produit fini en terme d'aspect et d'efficacité [PET 06]. La machine élimine les défauts inhérents à une production manuelle et permet à l'entrepreneur de se concentrer sur les défauts de conception en améliorant notamment l'articulation des deux branches et le maintien du ressort en position. D'ailleurs, nombre de brevets sont des pinces à linge améliorées (improved clothes-pin) comme celles de Smith de 1867 et 1874 et celles de Johnson, Boutell, Britton et Taylor A. L. vues plus haut.

\subsection{De la pince à linge au sèche-linge}

En 1920, l'entreprise Demeritt \& Palmer Packing Co. qui emploie 125 personnes fabriquant un demi-million de gross (58 cents la gross) de pinces à linge connues sous le nom de Holdfast se plaint de la concurrence de la Norvège et de la Suède en réclamant des mesures protectionnistes [DEM 21]. Des mesures seront prises en 1957 : la taxe douanière de 10 à 20 cents la gross, entraînant des protestations de la Suède, du Danemark et de la Belgique devant le GATT [EIS 57] : la pince à linge devient une affaire d'Etat... Les rapports se succèdent, en 1961 puis en 1962 et en 1978 [UST 61]. Malgré tous leurs efforts, les fabricants de pinces à linge du Vermont doivent jeter l'éponge avec la fermeture en 2003, dans un contexte de concurrence mondialisée, de la dernière entreprise, la National Clothespin Company [NAT 02]. Il existe encore aujourd'hui aux Etats-Unis des artisans qui poursuivent la fabrication sur commande de «pinces à linge américaines classiques » plus solides et résistantes, disent-ils, que les pinces à linge importées [CLA 20].

A partir du XXe siècle, la pince à linge en tant que dispositif de séchage du linge subit la concurrence du sèche-linge. Le français Jean-Marie Pochon obtient en 1799 [POC 99] un brevet de cinq ans pour des «buanderies communes à établir dans la capitale principalement pour l'hiver» en proposant un «ventilateur pour faire sécher le linge » entrainé manuellement avec une manivelle. Les 
premiers séchoirs électriques se diffusent aux Etats-Unis au sortir de la Seconde Guerre mondiale : au milieu des années 1950,10\% des Américains en étaient équipés, 78\% en 1997. En 2013, il y avait 89 millions de sèche-linges, peu performants, aux Etats-Unis (80\% des foyers en sont équipés) consommant environ 80 milliards de $\mathrm{kWh}$ par an [GRA 13]. En Europe, les sèche-linges sont moins gourmands et moins nombreux, mais consomment malgré tout quelques 30 milliards de kWh en 2020 [EUR 12].

Dans le nouveau contexte du changement climatique, la pince à linge prend une nouvelle jeunesse, synonyme de monde sans carbone, ustensile low-tech bien moins gourmand en énergie que le sèchelinge. Dans une campagne de publicité de 2007, EDF présente un visuel titré « Faisons le choix d'un monde sans $\mathrm{CO}_{2}$ » avec en premier plan une corde à linge et des pinces à linge et en second plan une femme rentrant son linge vers sa maison dans un paysage collinaire verdoyant [EDF 07] qui n'est pas sans rappeler le Vermont.

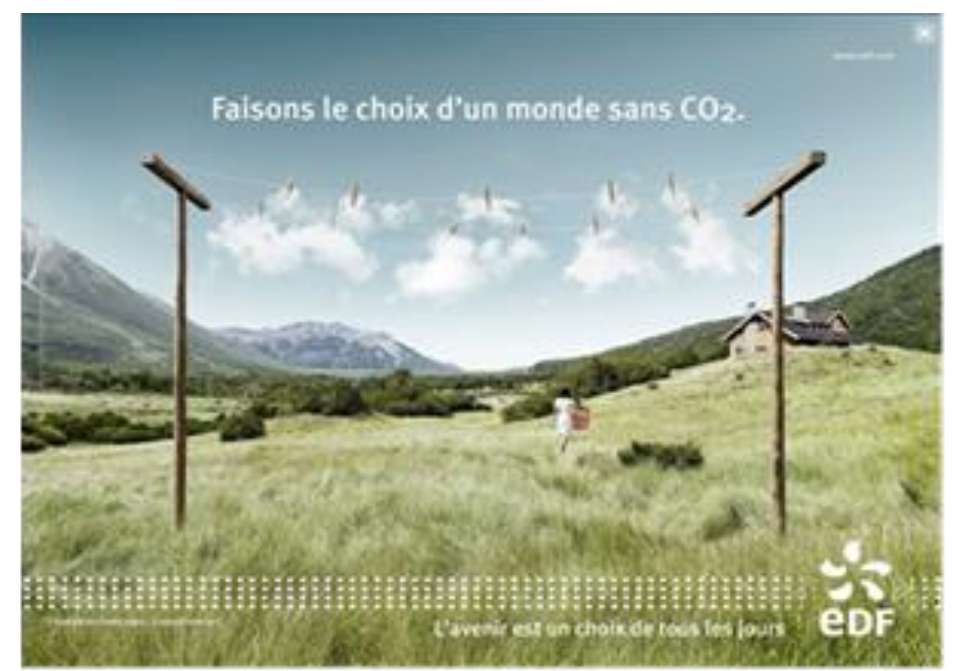

Figure 10. Visuel de la campagne de communication d'EDF en 2007.

Malgré cette concurrence du sèche-linge, le nombre de brevets de pince à linge est toujours resté important avec environ 100 brevets par décennie de 1955 à 2005 aux Etats-Unis et une nette recrudescence entre 2005 et 2015 liée à la problématique du changement climatique. En 150 ans, plus de 2000 brevets de pince à linge ont été déposés aux Etats-Unis...

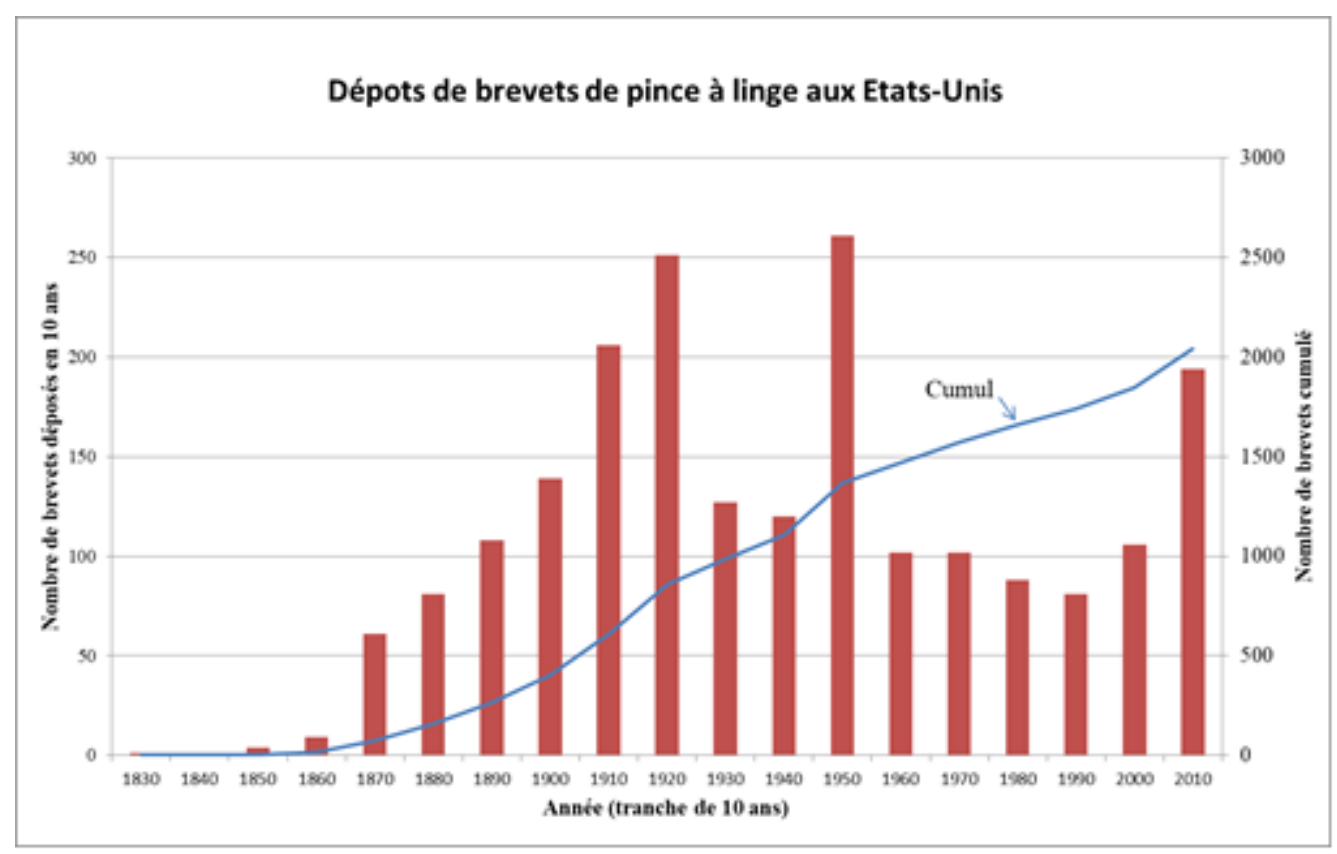

Figure 11. Nombre de brevets de pinces à linge aux Etats-Unis par décennie (1830-2010) [GOO 20]. 
En France l'entreprise Laguelle à Cosne d'Allier (Allier), petite entreprise de boissellerie fabriquant des pinces à linge en bois depuis 1946, lance au milieu des années 2000 la fabrication de pinces à linge brevetées par Barré et Lepage [BAR 05]. La fabrication de cette pince à linge utilise un procédé d'injection plastique bi-matière (polypropylène), une matière « dure » pour les branches et une matière «souple » pour la partie en contact avec le linge. Et ces deux matières peuvent avoir des couleurs différentes pour les rendre plus attractives. Lors du dépôt de ce brevet aux Etats-Unis, l'examinateur américain, Victor Sakran, ajoute comme référence, non sans malice, le brevet de D.M. Smith de 1853 : il faut bien convenir que le ressort de compression (et non plus de torsion comme celle de Moore, 1887) est proche de celui de Smith. 150 ans après David M. Smith, la pince à linge retrouve son ressort de compression, utilise de nouveaux matériaux, prend des couleurs, mais reste toujours identique à elle-même.

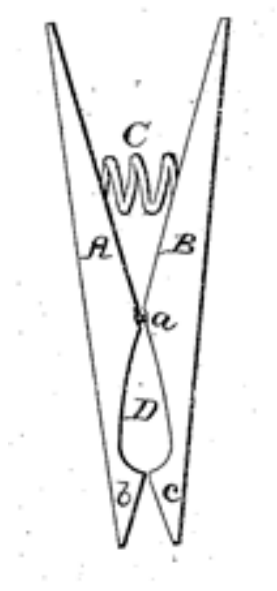

Smith, 1853.

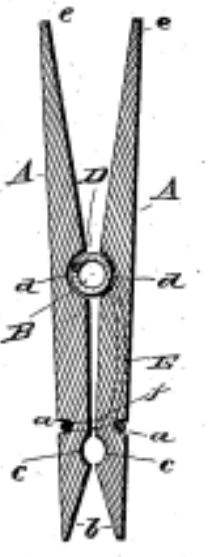

Moore, 1887.

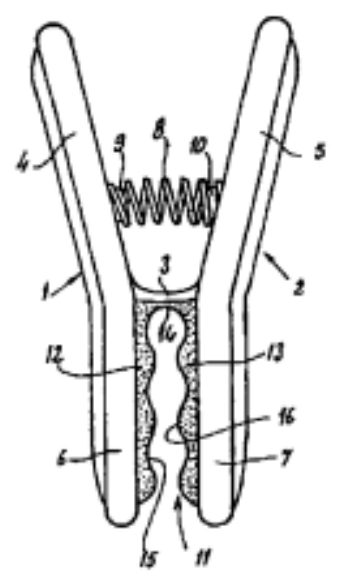

Barré et LePage, 2005.

Figure 12. 150 ans de pinces à linge.

Pour le designer lyonnais Bernard Barré, la pince à linge est un sujet idéal : un très beau marché avec des ventes s'élevant à 2,5 pinces à linge par an et par personne, un produit dont personne ne connaît exactement le prix de vente, un marché sans marques qui se bat sur les prix, un laissé pour compte dans le processus du traitement du linge : nous disposons de machines à laver sophistiquées, de lessives performantes et en fin de ce processus « des traces à chaque emplacement de pinces ! ». [BAR 09]

En écho à la situation vécue par D.M. Smith, Jean-Claude Laguelle, dirigeant du fabricant éponyme de pinces à linge, témoigne en 2011 «Oui, on peut exporter jusqu'au Japon des cintres et des pinces à linge produits en France. Plus précisément, à Cosne d'Allier, 2200 habitants, au cœur du bocage bourbonnais, pas bien loin de Montluçon [LAG 11]. » Du Vermont à l'Auvergne...

\section{La pince à linge, une icône américaine}

Dès 1853 , l'année même du brevet de D.M. Smith, la nation américaine s'affiche comme une nation d'inventeurs avec l'Exposition de l'industrie de toutes les nations (Crystal Palace Exhibition) de New York présentant au grand public les produits les plus divers de 1'«ingéniosité Yankee ». Deux décennies plus tard, en 1874, le public découvre au Musée des Modèles de l'Office des brevets [KEI 74] près de 10000 modèles d'inventions, des maquettes que devaient fournir les inventeurs à l'appui de leur demande de brevet.

En 1986 le Musée national d'histoire américaine organise une exposition intitulée «Demande de brevets : modèles d'invention » à l'occasion du $150^{\text {ème }}$ anniversaire du Patent Act de 1836. Le livret de cette exposition «Icones de l'invention - Modèles de brevet américain » (Icons of Invention American Patent Models) fait explicitement référence à la dimension iconique de ces inventions 
[JAN 90]. Les objets exposés sont des maquettes, le plus souvent fonctionnelles, que les demandeurs de brevets devaient fournir à l'appui de leur demande de brevet. Parmi les 113 modèles présentés, seuls deux d'entre eux sont déclinés en plusieurs exemplaires : neuf pinces à linge et quatre lampes à incandescence d'Edison. Douze ans plus tard, le même musée, le Smithsonian Institute à Washington, organise une exposition sur l'histoire des pinces à linge intitulée America's Clothespins présentant 41 modèles de pince à linge sur la période de 1852 à 1887 . La pince à linge entre dans l'histoire à une époque où elle disparaît de la mémoire collective : la conservatrice, Barbara Janssen, raconte qu'un jour où elle se promenait dans le musée, elle vit un jeune garçon se tourner vers son père et lui demander ce qu'était une pince à linge...

La pince à linge s'érige ainsi en icône de l'Amérique, plus précisément celle des pionniers, des territoires ruraux souvent dévalorisés par les grands centres urbains (le Vermont vu de New York...), d'une Amérique perdue, épisode fugace entre utopie agrarienne des origines et grande industrie moderne. C'est aussi une image romantique, une belle histoire, celle des entrepreneurs qui écrivent l'histoire sociale avec de petits objets insignifiants, sans prétention, mais qui font sens et trouvent leur clientèle.

\section{Conclusion}

D.M. Smith est à la fois inventeur et entrepreneur. Certaines de ses inventions donnent lieu à des entreprises, d'autres non ; certaines sont des échecs (serrures, paniers), d'autres des réussites éclatantes (pinces à linge, agrafes). D.M. Smith ne travaille pas seul, il agit au sein d'un groupe d'entrepreneurs et/ou d'inventeurs présents à Springfield. Certains sont plus inventifs, d'autres plus entreprenants, d'autres plus opportunistes ou suiveurs. Ce groupe fait société, une société de techniciens développant des produits tous azimuts pour des marchés hypothétiques dans une société américaine qui se cherche, entre âge d'or et nouvel âge doré (gilded age) pour reprendre Mark Twain [TWA 73].

Cette trajectoire est le déroulement d'un processus que D.M. Smith manifeste dès ses plus jeunes années, se renforce dans l'apprentissage du métier de charpentier, pour développer une solution nouvelle au maintien du linge. Comme l'écrit Samuel Smiles en 1861, les techniciens de la trempe de D.M. Smith «ont rassemblé leurs connaissances pratiques dans l'atelier, ou les ont acquises dans le travail manuel. Ils sont devenus célèbres, principalement par leurs habitudes d'observation, leurs pouvoirs de discrimination, leur perfectionnement constant et leur patiente industrie. » [SMIL 61].

Cette invention est d'abord fabriquée de façon artisanale, mise sur le marché et rencontre un grand succès. La mécanisation permet d'augmenter la production en diminuant les coûts, mais limite aussi les imperfections de la fabrication à la main. Dès lors, la pince peut être améliorée en fonction des retours d'expérience dans une boucle vertueuse invention/fabrication/amélioration.

Dans le contexte tumultueux des années 1840 , « le choix le plus sûr est d'innover, la technique est reine », comme le disait Braudel [BRA 92] et c'est le choix que fait D.M. Smith. Révélateurs de relations sociales [GAR 99], moteurs de la démocratie [BURLI 40], les objets techniques, notamment les objets du quotidien les plus courants, construisent une nouvelle société en résonance avec l'émancipation des femmes et la nouvelle «économie domestique » prônée par Beecher, avec l'affirmation de la mythique « ingéniosité Yankee » mécanisant les processus de fabrication, mais aussi la vie quotidienne et l'exploitation de nouvelles « conditions de Frontière » (énergie hydraulique, bois). Caractéristique de cette période révolue, la pince à linge se construit comme icône américaine, une sorte de «canon culturel » au sens d'identité nationale, mais aussi de nostalgie du temps des pionniers. 


\section{Bibliographie}

[ALD 52] AldRICH S., Clothes-pin, US Patent No 9,257, Sep. 14, 1852 ; Johnson C. C., Improved clothes-pin, US Patent No 63,393, Apr. 2, 1867 ; Boutell H. T., Improved clothes-pin, US Patent No 68,161, Aug. 27, 1867 ; BRITTON R. G., Improved clothes-pin, US Patent No 69,398, Oct. 1, 1867 ; TAYLOR A. L., Improved clothes-pin, US Patent No 76,547, Apr. 7, 1868.

[ALD 69] AlDRICH L. C. et Holmes F. R., History of Windsor county, Vermont, D. Mason \& Co., Syracuse, N. Y., 1869, p. $456-457$.

[ANO 00] «Clothespin facts », Portand Daily Press, August 16, 1900, p. 4.

[ANO 04] « Motive powers », Greater Vermont, The Vermonter, Vol. IX, No. 6, Jan. 1904, p. 27-28.

[ANO 15] «Making of clothes-pin - Humble Article Valuable By-product of Hardwood Mills », The Bamberg Herald, 25 Nov. 1915, p. 12.

[ANO 31] «Das große Waschfest vor der Stadt», dans Splendor solis (manuscrit alchimiste), Nüremberg, 1531. Source : Wikimedia Commons.

[BAR 05] BARRE B., LePAGE F., Clothes peg, US Patent No 6,842,951 B1, Jan. 18, 2005.

[BAR 09] BARRÉ B., All you need is L.O.V.E. (Live the Oblique Vision Experience) - Comment se réinventer sur des marchés saturés, Lyon, Mag2Lyon, 2009, p. 224.

[BEE 43] BEECHER C. E., A treatise of domestic economy, for the use of young ladies at home and at school, Boston, Thomas H. \& Co., 1843; ROBERTS Erie M., «Architecture of the Millennium: Catharine Beecher, Domestic Economy, and Social Reform », Constructing the Past, Vol. 7, Iss. 1, Article 5, 2006.

[BLA 10] «Black River and Falls at Springfield». Carte postale publiée vers 1910. Source : https://en.wikipedia.org/wiki/Springfield,_Vermont\#/media/File:PostcardSpringfieldVTBlackRiverAndFalls1910.jpg

[BRA 92] BRAUDEL Fernand, Civilisation matérielle, économie et capitalisme, XVe-XVIIIe siècle. 1 - Les Structures du quotidien, Paris, Armand Colin, 1992 [1979], p. 382.

[BUR 29] BUREAU OF LABOR STATISTICS, History of Wages in the United States From Colonial Times to 1928, Bulletin of the United States Bureau of Labor Statistics, No. 499, 1929, p. 160.

[BURLE 86] BuRLeigh L. R., Springfiel Vt, Published and drawn by L.R. Burleigh, Troy, N. Y., 1886.

[BURLI 40] Burlingame R., Engines Of Democracy - Inventions and Society in Mature America, Charles Scribner's Sons, New York, London, 1940, p. 4.

[CLA 20] Classic American Clothespins, Moravia, New York. Site Internet, consulté le 15 septembre 2020: http://classicamericanclothespins.blogspot.com/.

[COOL 59] CoOlidge A. J. et MANSFiEld J. B., History and description of New England, general and local, Austin J. Coolridge, Boston, 1859, p. 405.

[CRA 43] CRAIN E., Apparatus for the cure of curved spine, hernia, \&c., US Patent No 2,913, 16 Jan. 1843 (antedated 16 July, 1842).

[DEM 21] DEMERITT B. R., «Clothespins », Hearings of General Tariff Revision, Paragraph 176, Government Printing Office, Washington D.C., 1921 p. 1218-1221.

[EDF 07] EDF [Electricité de France], Faisons le choix d'un monde sans CO2, Visuel de la campagne L'avenir est un choix de tous les jours, Euro RSCG C \& O, 9 Mars 2007.

[EIS 57] EISENHOwER D. D., Withdrawal of Trade Agreement Concession on String Clothespins, No 3211, Nov. 1957 ; GATT [General Agreement on Tariffs and Trade], Summary of the twenty-first meeting, GATT, SR.12/21, dec., 11 1957, p. 178-181.

[ELL 57] ELLIS J. A. H., Form or mold in which wooden slats, etc, are made into baskets, US Patent No 16,953, Mar. 31, 1857.

[EME 12] EMERSON R. W., Journals, Boston, Houghton Mifflin, 1912, vol. 8, p. 234. Cité dans WaITE David Hawkes, Vermont in the Union: a study of the growing influence of the concept of Union in Vermont rhetoric, 1840 to 1861, Northwestern University, Evanston (Illinois), 1973.

[EUR 12] EUROPEAN COMMISSION, Impact assessment, Accompanying the document Commission Regulation implementing Directive 2009/125/EC of the European Parliament and of the Council with regard to ecodesign requirements for household tumble driers, Commission staff working document, Bruxelles, 2012 p. 8.

[FUL 45] FULLER M., Woman in the nineteenth century, Greeley \& McElrath, New York, 1845. 
[FULL 94] FULLER L. K., «Vermont inventors», dans UlLERY Jacob G. (dir.), Men of Vermont: An illustrated biographical of Vermonters and sons of Vermont, Transcript Publishing Company, Brattleboro, Vt., 1894, p. 190-196, p. 195.

[GAR 99] GARABUAU-MoussaOUi I. et DesjeuX D., Objet banal, objet social - Les objets quotidiens comme révélateurs des relations sociales, L'Harmattan, Paris, 1999.

[GIE 80] GIEDION Siegfried, La mécanisation au pouvoir, Centre Georges Pompidou/CCI, Paris, 1980.

[GOO 20] GOOGLE PATENTS, «Clothes peg», D06F55/00, Cooperative Patent Classification (CPC). https://patents.google.com/, recherche effectuée en Août 2020.

[GRA 13] GRANDA C., Bush E. et Wold C., «Market transformation for clothes dryers: lessons learned from the European experience », ECEEE 2013 Summer Study-RethinK, Renew, Restart, p. 524-527.

[GRE 12] GREENBAUM H. et WILSON C., « Who made that clothespin? », The New York Times Magazine, May 13, 2012, p. 21.

[HAY 81] HAYWARD S., History of the town of Gilsum, New Hampshire from 1752 to 1879, J. B. Clarke, Manchester, N.H., 1881.

[HUB 95] HubBard H. C. and DARTT J., History of the Town of Springfield 1752-1895, Vermont, G.H. Walker \& co., Boston, 1895.

[HUN 79] HUNTER L. C., Waterpower in the Century of the Steam Engine, First Ed., Published for the Eleutherian MillsHagley Foundation by the University Press of Virginia, Charlottesville, 1979.

[JAB 14] JABLONKA I., L'histoire est une littérature contemporaine - Manifeste pour les sciences sociales, Paris, Seuil, 2014.

[JAN 90] JANSSEN B. S., Icons of Invention - American Patent Models, National Museum of American History and Smithsonian Institution, Washington D.C., 1990.

[KAU 97] KaUfMANn J.-C., Le cour à l'ouvrage - Théorie de l'action ménagère, Nathan, Paris, 1997.

[KEI 74] KeIM De B., Keim's illustrated guide to the museum of models, Patent office, DeB. Randolph Keim, Washington, D.C., 1874.

[KER 76] KeRBER L., «The Republican Mother: Women and the Enlightenment-An American Perspective », American Quarterly, 1976, vol. 28, no 2, p. 187-205.

[LAC 18] LACROIX J.-M., Histoire des États-Unis, 6ème éd., PUF, Paris, 2018, p. 207.

[LAG 11] LAGUELLE J.-C. «La pince à linge Made in Auvergne », témoignage d'acteur dans PoRTNOFF André-Yves, « Révolution de l'intelligence : acteurs et facteurs », Futuribles, 2011, No 374, p. 46.

[LAH 07] LAHEY A., «Clothespin - in search of the perfect pegs », RD, Aug. 2007, p. 48.

[MAS 59] Mason A. C., Clothes Pin Machine, US Patent No 25,344, Sep. 6, 1859.

[MAY 81] MAYALL D., Itinerant minorities in England and Wales in the nineteenth and early twentieth centuries - A study of gypsies, tinkers, hawkers and other travellers, Ph. D., University of Sheffield, 1981. p. 109-111

[MILL 10] MiLler M. S., Inspired Innovations: A Celebration of Shaker Ingenuity, University Press of New England, Hanover and London, 2010, p. 184 et p. 189.

[MOO 87] MoORE S. E., Clothes Pin, US Patent No 365,755, Jun. 28, 1887.

[MOR 81] MORISOT B., La blanchisseuse ou femme étendant du linge, 1881. Source : Wikimedia Commons.

[MUN 64]. MunN \& Co., The United States Patent Law. Instructions How to Obtain Letters Patent for new inventions, New York, Munn \& Co., 1866, p. 9.

[NAT 02] NATIONAL Clothespin FACtORY, Montpelier, Washington County, Vermont, National Register of Historic Places, United States Department of the Interior, Washington D.C., 2002.

[NYE 98] NyE D. E., Consuming Power: A Social History of American Energies, The MIT Press, Cambridge, Mass., 1998, p. 54.

[PET 06] Petroski H., «Engineering: Things Small and Large », American Scientist, Vol. 94, No. 3, 2006, p. $212-215$.

[PIE 57] PIERPONT Rev. J., «Whittling-A Yankee Portrait, », United States Magazine, 4, 1857, p. 217. Cité dans : COOPER C. C., «Myth, Rumor, and History - The Yankee Whittling Boy as Hero and Villain », Technology and Culture, Vol. 44, 2003, p. 82-96. 
[POC 99] POCHON J.-M., Buanderies communes à établir dans la capitale principalement pour l'hiver, Brevet d'invention de 5 ans 1BA102, déposé le 18 Thermidor an 6 [6 août 1798]. Source: INPI, http://basesbrevets19e.inpi.fr/

[PRI 32] PRIOR S., Device for fastening clothes on a line, US Patent No -6973, Mar. 22, 1832.

[RIC 00] Richardson F. W., Nineteenth Century Springfield - From an agricultural to industrial community, Springfield Printing Corporation, Springfield, 2000.

[RID 29] RIDER A., Camp meeting - 1820-1830, Kennedy \& Lucas's Lithography, 1829. Source : Library of Congress, Washington, D.C. https://www.loc.gov/item/96510018/

[RIF 80] RifFault M.-C., "De Chaptal à la Mère Denis: Histoire de l'entretien du linge domestique », Culture Technique, 1980, nº 3, p. 257-263, p. 263.

[ROC 97] ROCHE D., Histoire des choses banales. Naissance de la consommation, XVIIème-XIXème siècle, Fayard, Paris, 1997.

[ROE 26] RoE J. W., English and American tool builders, New York, London, McGraw-Hill, 1926 [1916], p. $199-201$.

[SAN 85] SANBORN FIRE InSURANCE, Sanborn Fire Insurance Map from Springfield, Windsor County, Vermont, Sanborn Map Company, 1885, 1894, 1900, 1905 and 1910. Source : Library of Congress, Washington, D.C.

[SCH 94] SCHNIETZ K. E., «The 1916 Tariff Commission: Democrats' Use Of Expert Information to Constrain Republican Tariff Protection », Business and Economic History, vol. 23, n 1, 1994, p. 176-189.

[SEA 12] SEARS, RoebuCK AND Co., Catalog no. 124, Chicago, 1912, p. 1020.

[SEC 07] SECTER B., «Springfield runner-up in worst... vote... ever », Chicago Tribune, July 11, 2007. Le vote était organisé par le journal USA Today et a connu un grand succès avec près de 110000 votes. Grâce à la mobilisation de ses 9000 habitants, Springfield, Vermont l'emporte de 733 voix devant Springfield, Illinois, une ville bien plus importante (110 000 habitants).

[SGB 40] S. G. B., «Pleasures of the factory life », The Lowell Offering: a repository of original articles on various subjects written by factory operatives, 1840, $\mathrm{N}^{\circ} 1, \mathrm{p} .25$.

[SMI 32] SMith D.M., «Awl-haft», US Patents No -7,263, Oct. 25, 1832 et No -9,576, Mar. 31, 1836. Cité dans : LEGGETT Mortimer Dormer (ed.), Subject-matter index of patents for inventions issued by the United States Patent office from 1790 to 1873, Government Printing Office, Washington D.C., 1874, vol. 1, p. 27. La numérotation des brevets commence en 1836, les brevets antérieurs ayant un numéro négatif ( $\mathrm{Nr}-1$ le 31 Juillet 1790).

[SMI 46] SMITH D.M., Permutation Lock, US Patent No 4,635, July 14, 1846 ; Method of preserving the shape of steel springs in the process of tempering, US Patent No 5,979, Dec. 19, 1848 ; Bank-Lock, US Patent No 6,272, Apr. 3, 1849 ; Sewing Machine, US Patent No 7,296, Apr. 16, 1850.

[SMI 53] SMITH D.M., Spring-clamp for clothes-lines, US Patent No 10,163, Oct. 25, 1853.

[SMI 54] SMITH D.M., Lathe-dog, US Patent No 10,939, May 16, 1854.

[SMI 59] SMith D.M., Improvement in seed-planters, US Patent No 23,955, 10 May 1859 ; avec WhiTMORE O. W., Carpenter's rule, US Patent No 25,460, Sep. 13, 1859 ; Hook and eye, US Patent No 28,443, May 22, 1860.

[SMI 63] SMITH D.M., Improvement in spring-hook fastenings for garments, US Patent No 38,920, Jun. 16, 1863 ; Paper Holder, US Patent 58,687, Oct. 9, 1866.

[SMI 67] Improved clothes-pin, US Patent No 63,759, Apr. 9, 1867 ; Improvement in clothes-pins, US Patent No 150,439, May 5, 1874.

[SMIG 80] SMITH G., Gipsy Life, being an account of our Gipsies and their children,London, Haughton and Co., Londres, 1880.

[SMIL 61] SMILES S., Lives of the engineers, Murray, Londres, 1861, p. xvii.

[SPR 83a] SPRINGFIELD Downtown Historic District, «\# 25. The David M. Smith House, 138 Main Street: (c.1858) », National Register of Historic Places Inventory, United States Department of the Interior, 1983, p. 15.

[SPR 83b] SPRINGFIELD DownTOwn Historic DisTRICT, «David M. Smith House from Main Street - Photograph \# 39 », National Register of Historic Places Inventory, United States Department of the Interior, 1983, p. 272.

[THE 20] ThE ChuRch OF Jesus-Christ OF LATTER-DAY SAINTS, Pioneer Database 1847-1868, 2020. https://history.churchofjesuschrist.org/overlandtravel/pioneers/53703/luther-singleton-hemenway

[THO 54] THOREAU H. D., Walden ou La vie dans les bois, Gallimard, Paris, 1990 [1854]. 
[TUC 43] TUCKER G., Progress of the United States in population and wealth in fifty years as exhibited by the decennial census, Press of Hunt's Merchant's Magazine, New York, 1843, p. 57.

[TUR 20] TURNER F. J., The frontier in American history, H. Holt and Company, New York, 1920, p. 78.

[TWA 73] TwaIn Mark, Warner Charles Dudley, The Gilded Age, American Publishing Company, San Francisco, 1873.

[USP 69] USPTO Patent Office, Annual report of the Commissioner of Patents, Washington, Goverment Printing Office, 1869 , vol.1, p. 9.

[UST 61] USTC [United States Tariff Commission], Spring clothespin, Report to the President, TC Publication 43, USTC, Washington, 196 ; Standard clothespin, Report on Escape-Clause Investigation No. 7-110, TC Publication 50, USTC, Washington, 1962 ; Clothes pins, Report to the President on Investigation No. TA-203-36 Under Section 201 of the Trade Act of 1974, USTC, Washington D.C., 1978.

[VER 10] Vermont Wood Products MARKeTing Council, History of Woodworking in Vermont, 1791 - Today, 2010.

[VES 44] VESEY F. B., Gypsies of Britain, Chapman \& Hall, Londres, 1944, p. 193-197.

[WAR 80] WARD D., Religious enthusiasm in Vermont, 1761-1847, Ph.D. Theology, University of Notre Dame, Notre Dame (Indiana), 1980.

[WEL 19] Welch R. et LAMPHIER P. A. (eds.), Technical Innovation in American History: An Encyclopedia of Science and Technology, ABC-CLIO, Santa Barbara, California, 2019, vol. 1, p. 196-197. 